Maciej Różewicz

\title{
Shape Optimization of a Flywheel
}

\section{Introduction}

A flywheel is quite a simple tool (if it comes for idea of working), used for storing energy. It is stored in the form of kinetic energy of a rotary mass. Thus, the maximum energy stored in a flywheel is given by:

$$
E_{\text {kmax }}=\frac{1}{2} J \omega_{\max }^{2}
$$

where:

$J$ - moment of inertia of flywheel,

$\omega_{\max }-$ maximum rotary speed,

$E_{\text {kmax }}$ - maximum stored energy.



Fig. 1. Schematic diagram of a commercial flywheel system [10]

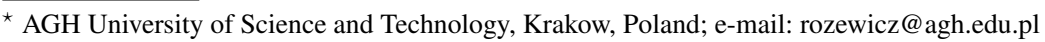


However, for practical reasons flywheel systems (Fig. 1) are not completely discharged, it is not cost-effective due to the existence of large losses during the ramp-up from zero velocity.

Typically they are discharged to a certain minimum value $\omega_{\min }$, selected such that the $\frac{\omega_{\min }}{\omega_{\max }}=s=0.25$ [5] - this selection of minimum speed value provides use about $94 \%$ of maximum stored energy $E_{k \max }$. So the maximum power delivered to the receiver can be expressed as:

$$
\Delta E_{\text {kmax }}=\frac{1}{2} J\left(\omega_{\max }^{2}-\omega_{\min }^{2}\right)=\frac{1}{2} J \omega_{\max }^{2}\left(1-s^{2}\right)
$$

Of course, this situation depicted by equation (2) is quite idealized. In reality, one must take into account the losses of energy occurring in the system. They are caused by a number of factors: friction in bearings, aerodynamic friction, inducing forces in the coils as well as the efficiency of the power converter, etc. In this case, equation (2) should be supplemented by an efficiency factor $\eta(0<\eta<1)$.

$$
\Delta E_{k \max }=\frac{1}{2} \eta J \omega_{\max }^{2}\left(1-s^{2}\right)
$$

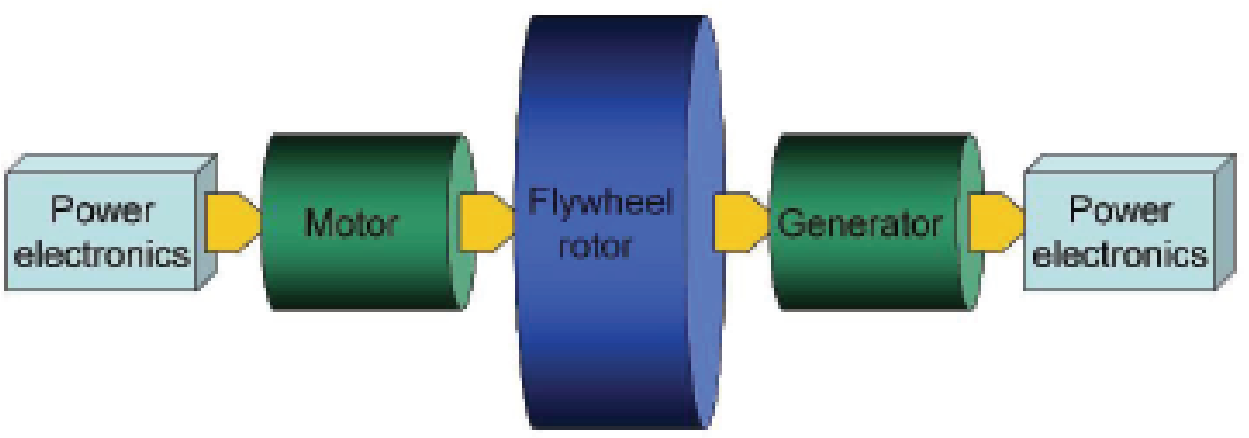

Fig. 2. Schematic flow of energy in a flywheel accumulator [10]

On the basis of the above depending for the energy accumulated and transmitted to the load can be seen that in order to increase it, can increase the flywheel moment of inertia or increase rotary speed. It is also seen that increasing the speed gives a much higher increase in the energy accumulated, as energy is proportional to the square of speed, and depends only linearly to moment of inertia.

That is why trying to design flywheels for high speed (as far as possible, not always possible to obtain a sufficiently high turnover due to occur over time stress), which allows to obtain the same amount of energy accumulated in smaller geometric dimensions, lower 
weight. Describing the size of the ratio of the maximum accumulated energy to volume (mass) is called the energy density (specific energy) and is given by the volume:

$$
e_{k, v}=\frac{E_{k \max }}{V}=\frac{J \omega_{\max }^{2}}{2 \int d V}
$$

and for mass:

$$
e_{k, m}=\frac{E_{k \max }}{m}=\frac{J \omega_{\max }^{2}}{2 \rho \int d V}
$$

where:

$e_{k, v}$ - energy density,

$e_{k, m}$ - specific energy,

$V$ - volume of flywheel,

$m$ - mass of flywheel.

The maximum specific energy can also be expressed using the ratio of the maximum stress resistance and density of the material from which the rotor is done. Then $e_{k, m}$ is directly proportional to it with an appropriate scaling factor $K$, which depends only on the shape of the flywheel (equation (6)).

$$
\hat{e}_{k, m}=K \frac{\sigma_{\theta \max }}{\rho}, \hat{e}_{k, v}=K \sigma_{\theta \max }
$$

Tables of coefficients for the different shapes can be found for example in [5].

Providing maximum energy density of the battery makes it possible to minimize the dimensions and weight. Small size and weight allow, in addition to reducing the space required for the batteries and e.g. placing a battery in vehicles. What is already being done by manufacturers such as Porsche, Jaguar and Formula 1 racing cars.

The parameters presented here (maximum accumulated kinetic energy, moment of inertia, energy density) are essential to determine the quality of the flywheel as the energy storage battery. During the design process, you can try to optimize them. The evaluation criterion can be formulated in various ways, this may be the optimization of one selected parameter or multi-criteria optimization seeking to choose the best combination of all parameters.

In this work will be presented approach to maximize the moment of inertia of the flywheel and at the same time increasing energy density. Imposed will be appropriate limitations related to the strength of the material from which it is made. Calculations are performed for the construction of the rotor in the shape of the pierced cylinder (shown in Fig. 3). For such a shape can be obtained analytical depending on stresses arising during work time. 

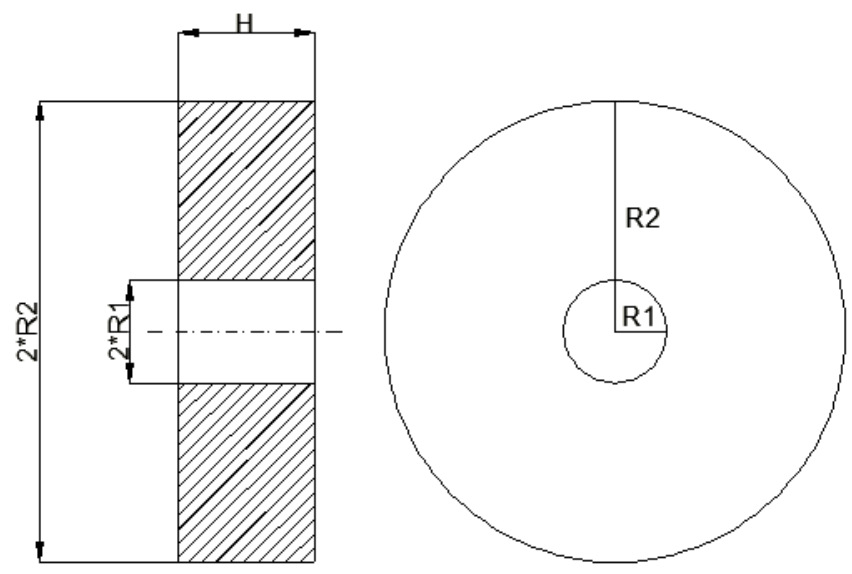

Fig. 3. Top view and cross-section of the flywheel in the shape of a cylinder

In the case under consideration the shape of the moment of inertia depends linearly on the height, but specific energy $e_{k, m}$ does not depend from it. Therefore, you can pretty well compare to what size the size of the optimal values take on the plane $R_{1}-R_{2}$. This is shown in the drawing (Fig. 4). It may be noted here that these are largely conflicting requirements, the maximum moment of inertia is achieved when the shape is similar to a fully filled roller, while the maximum power density is obtained close to the ideal shape of the ring. These conflicting requirements suggest the use of multi-criteria optimization to determine the best shape.
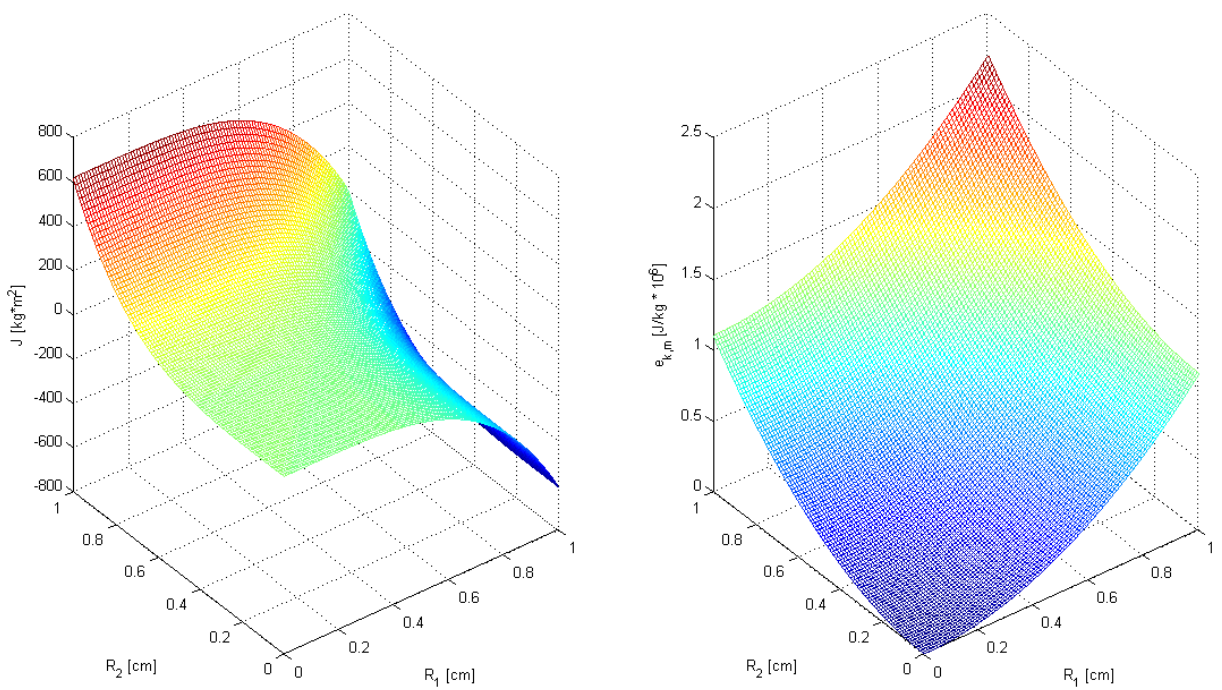

Fig. 4. Comparison of the formation of the moment of inertia (left) and energy density $e_{k, m}$ depending on the radii $R_{1}$ and $R_{2}$ 


\section{Moment of inertia}

The moment of inertia is a measure of inertia of the body in rotation. The bigger it is, the harder the body is to disperse or stop. As it has been mentioned in the preceding paragraph, moment of inertia has an influence on kinetic energy accumulated in the rotating block.

$$
J=\iiint\left(x^{2}+y^{2}\right) \rho(x, y, z) d x d y d z
$$

where $\rho(x, y, z)$ - material density in point $(x, y, z)$.

In practice, however, it can be assumed that the entire volume of the solid material density is constant. Additionally you can use designation $x^{2}+y^{2}=r^{2}$ (square of the distance from the axis of rotation). Then, the equation (7) takes the simpler form (8):

$$
J=\int r^{2} \rho d V=\int r^{2} d m
$$

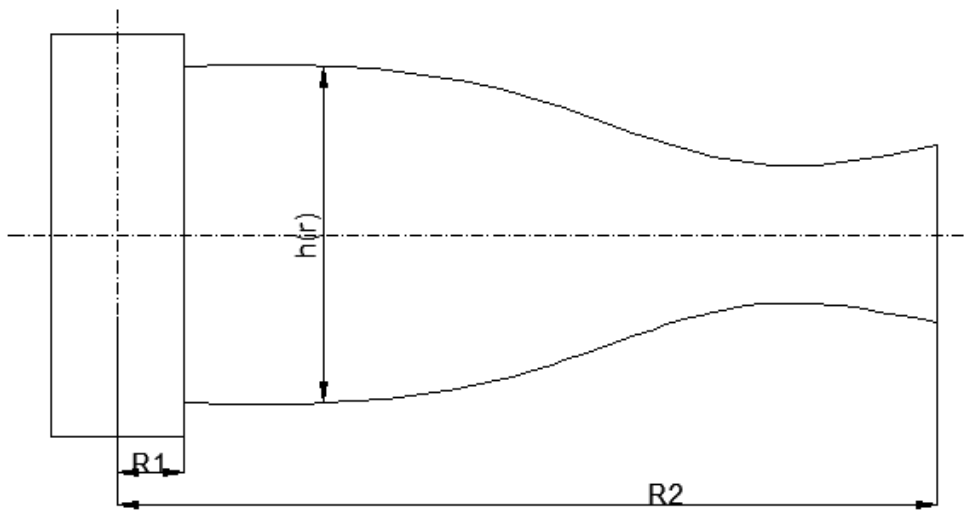

Fig. 5. Half of the sample cross-section shape of the flywheel

In the case of less general, when the rotational body is symmetrical relative to the radius, as shown in Figure 5, and such is the form used by the majority of practical flywheels, the moment of inertia takes form:

$$
J_{S}=2 \rho \pi \int_{R_{2}}^{R_{2}} r^{3} h(r) d r
$$

where:

$h(r)$ - section height as a function of distance from the axis of rotation, $R_{1}, R_{2}$ - respectively initial and final distance of cross-section from axis of rotation. 
In the case of the considered shape of the flywheel, the expression on their moment of inertia takes the already much simpler form:

$$
J_{C}=\frac{1}{2} \rho \pi H\left(R_{2}^{2}-R_{1}^{4}\right)
$$

Notation as shown on Figure 3.

\section{Stresses}

During operation of the flywheel, as a result of the rotational speed, and more specifically the centrifugal forces occurring, stresses arise in the rotor. There are two types of stress there occurs:

- tangential stresses $\sigma_{\theta}$,

- radial stresses $\sigma_{r}$.

a)

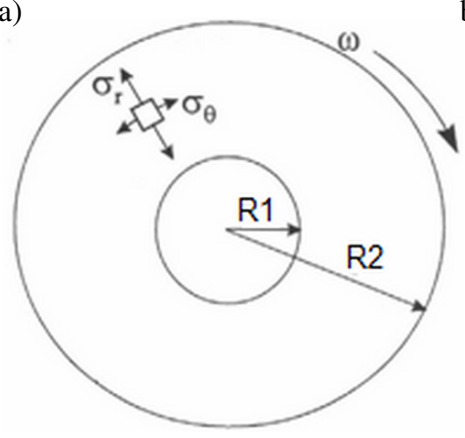

(a) b)

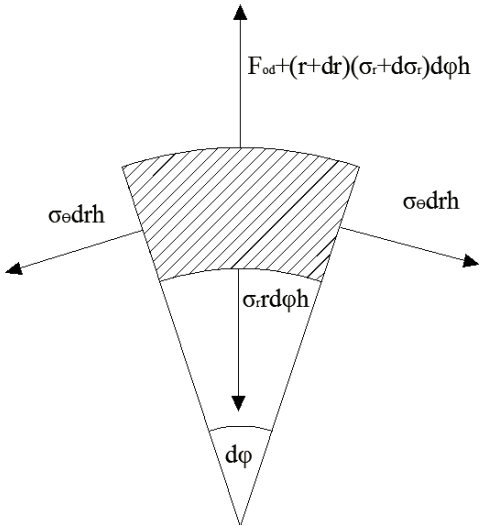

(b)

Fig. 6. Propagation of stress in the flywheel

The mechanism of their formation and the forces that cause them are marked on the Figure 6. On the basis of it, the formula of the centrifugal force can be presented:

$$
F_{C}=\rho h r^{2} \omega^{2} d \phi d r
$$

where $h=h(r)$ is the thickness of the flywheel depending on the distance from the axis of rotation.

And comparing the sum of the forces on the axis of a "vertical" in the Figure $6 \mathrm{~b}$ to zero, the differential equation is obtained that describes the impact of stress on the selected section of material [1]:

$$
\rho h r^{2} d \phi d r \omega^{2}+(r+d r)\left(\sigma_{r} h+\frac{d}{d r}\left(\sigma_{r} h\right) d r\right) d \phi h-2 \sigma_{\theta} d r h \sin \frac{d \phi}{2}-\sigma_{r} r d \phi h=0
$$


Given that there is equality $\sin \alpha \approx \alpha$ for small $\alpha$ and dividing equation (12) by $d \phi$ is obtained a simplified form of the equation:

$$
\frac{d \sigma_{r}}{d r} h r+h\left(\sigma_{r}-\sigma_{\theta}\right)-h r^{2} \omega^{2} \rho=0
$$

In addition, if we apply the substitution $\Phi(r)=\sigma_{r} h r$, and using depending on:

$$
\left\{\begin{array}{l}
\sigma_{r}=\frac{\Phi(r)}{h r} \\
\sigma_{\theta}=\frac{1}{h} \frac{d \Phi(r)}{d r} \rho \omega^{2} r^{2}
\end{array}\right.
$$

equation is obtained with only one unknown $\Phi(r)\left(\right.$ instead of two $\left.\sigma_{r}, \sigma_{\theta}\right)$.

$$
r^{2} \frac{d^{2} \Phi}{d r^{2}}+r \frac{d \Phi}{d r}-\Phi+(3+v) \rho \omega^{2} h r^{3}-\frac{r}{h} \frac{d h}{d r}\left(r \frac{d \Phi}{d r}-v \Phi\right)=0
$$

In general, for any $h(r)$, the analytical solution of this equation does not exist. Only in the case where $h(r)=h=$ const it can give an explicit analytical solution (if the last component is reset to the left side of the equation (15) because it is multiplied by the derivative $d h(r) / d r$ ) for $\sigma_{r}$ and $\sigma_{\theta}$. These equations (16) are presented below, along with a comment. However, for shapes other than the cylinder is usually used Finite Element analysis. This approach is shown for example in $[1,2]$.

The solutions of equation (15) are presented in the work [3] and [4], they were presented for the case of isotropic materials, namely those where there is no difference in physical properties of the material regardless of the direction of measurement (such as steel or aluminum):

$$
\left\{\begin{array}{l}
\sigma_{i r}=\frac{3+v}{8} \rho \omega^{2}\left(R_{2}^{2}+R_{1}^{2}-\frac{R_{1}^{2} R_{2}^{2}}{r^{2}}-r^{2}\right) \\
\sigma_{i \theta}=\frac{3+v}{8} \rho \omega^{2}\left(R_{2}^{2}+R_{1}^{2}+\frac{R_{1}^{2} R_{2}^{2}}{r^{2}}-\frac{1+3 v}{3+v}\right) r^{2}
\end{array}\right.
$$

As seen from the given equations (16), the resulting stress does not depend on the thickness of the cylinder, but only the rays from the internal and external.

Calculations for anisotropic materials (disparities physical properties depending on the direction of measurement) are usually more complex and are carried out in the Finite Element Method. Therefore, they will not be carried out here. This information can be found in the works: $[4,5,6]$.

Of course, the flywheel must withstand the maximum resulting stress (the maximum allowable stresses for the materials selected shows), should therefore be determined maximums specified functions. 
In the case of isotropic materials find maximum analytical methods are quite simple and maximum points are as follows:

$$
\left\{\begin{array}{l}
r_{i r, \max }=\sqrt{R_{1} R_{2}} \\
r_{i \theta, \text { max }}=R_{1}
\end{array}\right.
$$

In Figure 7 are examples of courses and radial shear stress for isotropic materials. Additionally, the process flow presented in the maximum stress depending on the ratio $R_{1} / R_{2}=\lambda$ and the original set $R_{1}$.
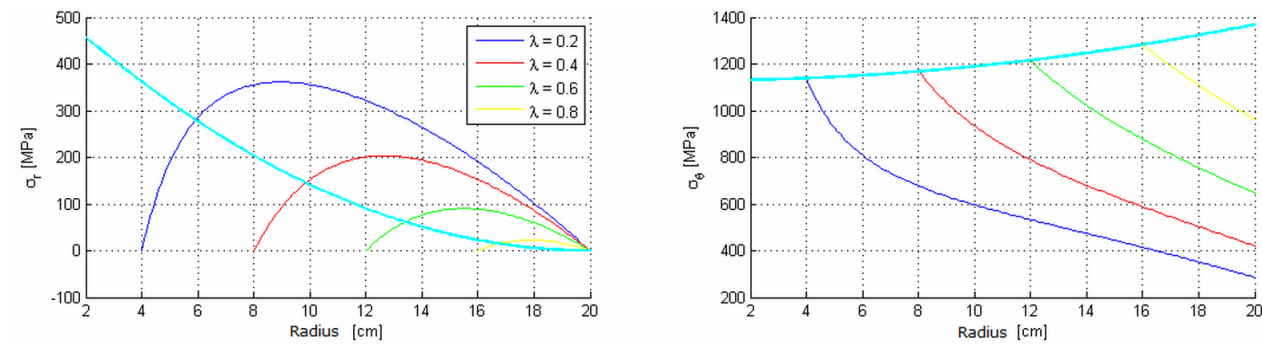

Fig. 7. Exemplary waveforms of radial stress (left) and tangential to the flywheel in the shape of a cylinder made of steel (isotropic). The bold line is the graph of $\sigma_{\max }(\lambda)$

\section{Formulation of optimization problem}

As indicated in the introduction, the task of optimizing the shape of the flywheel can be formulated in various ways. You can only maximize the moment of inertia of the relevant restrictions imposed on emerging during work stress and forces that can occur in the bearings due to the imbalance of the rotor as well as try to optimize the energy density at the same limitations, or create a multi-criteria optimization task of maximizing the moment of inertia (energy density) and minimize the stress occurring.

In this work it was decided to first of these approaches. So, to maximize the moment of inertia, the flywheel in the shape of a cylinder:

$$
\max _{R_{1}, R_{2}, H}\left\{\frac{1}{2} \rho \pi H\left(R_{2}^{4}-R_{1}^{4}\right)\right\}
$$

As well as specific energy, for the cylinder:

$$
\max _{R_{1}, R_{2}, H}\left\{\frac{1}{4} \omega_{\max }^{2}\left(R_{2}^{2}+R_{1}^{2}\right)\right\}
$$

Geometric constraints were imposed on the dimensions related to eliminate the possibility of setting the values so that the inner radius is greater than the outer radius. Also with the 
limitations associated with the maximum possible space activities. They take the form:

$$
\left\{\begin{array}{l}
H_{\text {min }} \leq H \leq H_{\text {max }} \\
R_{\text {min }} \leq R_{1} \leq R_{2} \leq R_{\text {max }}
\end{array}\right.
$$

Limitations on stress take the form of:

$$
\left\{\begin{array}{l}
\sigma_{r, \max } \leq \bar{\sigma}_{r, \max } \\
\sigma_{\theta, \max } \leq \bar{\sigma}_{\theta, \max }
\end{array}\right.
$$

As already mentioned in section 3 of the maximum stress can be determined in a simple manner for isotropic materials (from equations (16) and (17)):

$$
\left\{\begin{array}{l}
\bar{\sigma}_{r, \text { max }}=\frac{3+v}{8} \rho \omega_{\text {max }}^{2}\left(R_{1}^{2}+R_{2}^{2}-2 R_{1} R_{2}\right) \\
\bar{\sigma}_{\theta, \text { max }}=\frac{3+v}{8} \rho \omega_{\text {max }}^{2}\left(2 R_{2}^{2}+2 \frac{1-v}{3+v} R_{1}^{2}\right)
\end{array}\right.
$$

From the above equations can be determined by an equation showing explicitly the dependence $R_{2}\left(R_{1}\right)$ curves limiting the feasible solutions.

$$
\left\{\begin{array}{l}
R_{2}^{\sigma_{r}}=R_{1}+\sqrt{\frac{\bar{\sigma}_{r, \text { max }}}{\frac{3+v}{8} \rho \omega_{\max }^{2}}} \\
R_{2}^{\sigma_{\theta}}=\sqrt{\frac{\bar{\sigma}_{\theta, \max }}{\frac{3+v}{4} \rho \omega_{\max }^{2}}-\frac{1-v}{3+v} R_{1}^{2}}
\end{array}\right.
$$

For a more complete account of all the conditions to be met by the actual layout must take into account the reaction force generated in the bearings due to rotor unbalance. These forces can not be greater than the maximum strength of the applied bearings. Therefore, you should add another restriction:

$$
F_{R} \leq F_{R, \max }
$$

The reaction forces in the bearings may be calculated for example as in [7].

In the case described above, optimization was performed for a fixed rotational speed of the rotor. May also be considered optimization task where one of the decision variable is the rotational speed (with the proviso of course, from the top). Then it may be that a better solution would be to build a slower flywheel with a greater moment of inertia, thus a smaller stress in the present. You must also change the task of optimizing the moment of inertia for the task of minimizing the kinetic energy:

$$
\max _{R_{1}, R_{2}, H, \omega_{\max }}\left\{\frac{1}{4} \omega_{\max }^{2} \rho \pi H\left(R_{2}^{4}-R_{1}^{4}\right)\right\}
$$


In addition to limiting the top speed, the rest remains unchanged restrictions.

Since in the case considered the shape of these requirements are quite large extent conflicting. So to the final outcome of it was used to balance the multi-criteria optimization algorithm, maximizing vector:

$$
F\left(H, R_{1}, R_{2}\right)=\left[F_{1}\left(H, R_{1}, R_{2}\right), F_{2}\left(H, R_{1}, R_{2}\right)\right]^{T}
$$

where:

$F_{1}$ - moment of inertia,

$F_{2}$ - specific energy.

\section{Optimization results}

To solve the task was used to optimize the package MATLAB [8].

In the first place has been designated set in the plane of criteria $F_{1}-F_{2}$, which can be achieved with the constraints imposed on the job. It has been illustrated in Figure 8. The collection has been achieved marked by pointing, while the bold line has been selected Pareto set, ie a set dominated solutions (a set whose elements satisfying relationship $P=\{x \in U$ : $\forall y \in U[F(y) \geq F(x) \Rightarrow x=y]\}$, where $U$ is a set of parameters, which make the optimization). Material parameters were adopted in accordance with the Table 1, the other parameters are shown in Table 2.

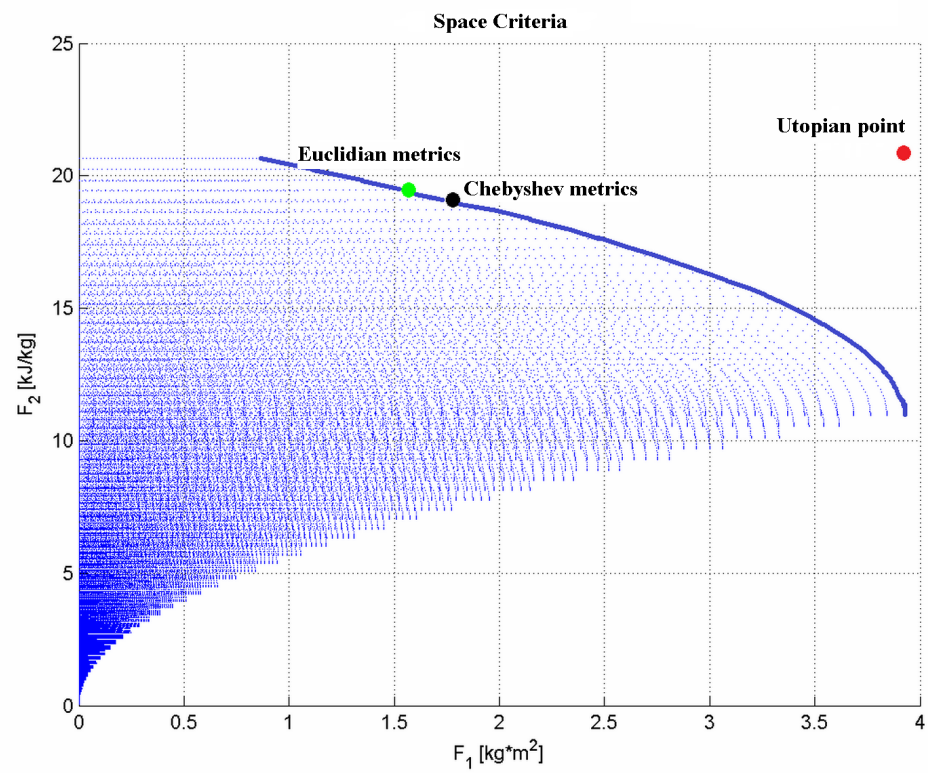

Fig. 8. Space criteria of optimal points marked 
Table 1

Used properties of selected materials [4]

\begin{tabular}{|c|c|c|c|c|c|c|}
\hline Material & $\rho\left[\mathrm{kg} / \mathrm{m}^{3}\right]$ & $E_{\theta}[\mathrm{GPa}]$ & $E_{r}[\mathrm{GPA}]$ & $\sigma_{\theta}[\mathrm{MPa}]$ & $\sigma_{r}[\mathrm{MPa}]$ & $e_{k, m}[\mathrm{~kJ} / \mathrm{kg}]$ \\
\hline Steel & 7800 & 200 & 200 & 800 & 800 & 51 \\
\hline Aluminum & 2700 & 70 & 70 & 500 & 500 & 93 \\
\hline Glass fiber & 2000 & 45 & 5 & 1000 & 40 & 250 \\
\hline Glass fiber & 1580 & 300 & 5 & 750 & 25 & 237 \\
\hline
\end{tabular}

Table 2

The values of the parameters adopted

\begin{tabular}{|c|c|c|c|}
\hline$\omega_{\max }[\mathrm{rpm}]$ & $H_{\max }[\mathrm{m}]$ & $R_{\max }[\mathrm{m}]$ & $F_{R, \max }[\mathrm{kN}]$ \\
\hline 10000 & 0,2 & 0,2 & 3 \\
\hline
\end{tabular}

Finding the optimal solution was made by scalarization by distance, as described for example in [9]. It involves the determination of the utopian, whose coordinates satisfy the relation:

$$
F_{i}^{u}=\max x \in U\left\{F_{i}(x)\right\}
$$

This is usually the point does not belong to the set attainable in the criteria, so it was in this case (red point in figure (8)) where the point was worth:

$$
F^{u}=[3,920720,863]^{T}
$$

The point considered as optimal point from the set of Pareto is located at a minimum distance from the point of utopian. Determination of this point depends on the selected metric. The study has been calculated for two cases:

- Euclidean metrics $-F_{e}=[1.569519 .4585]^{T}$ (green point on the graph),

- Chebyshev metrics $-F_{c}=[1.776019 .0768]^{T}$ (black point on the graph).

In the space of decision parameters correspond to the points:

$-X^{e}=[0.20000 .17600 .2000]^{T} \mathrm{mmm}$,

$-X^{c}=[0.20000 .17200 .2000]^{T} \mathrm{mmm}$.

Analogous calculations were performed for the situation where an additional decision variable was the maximum speed reached by the flywheel. Upper bound was $\omega_{\max }=40000 \mathrm{rpm}$. Again, was designated collection available in the parameter space and a set of Pareto and utopian point (8). 


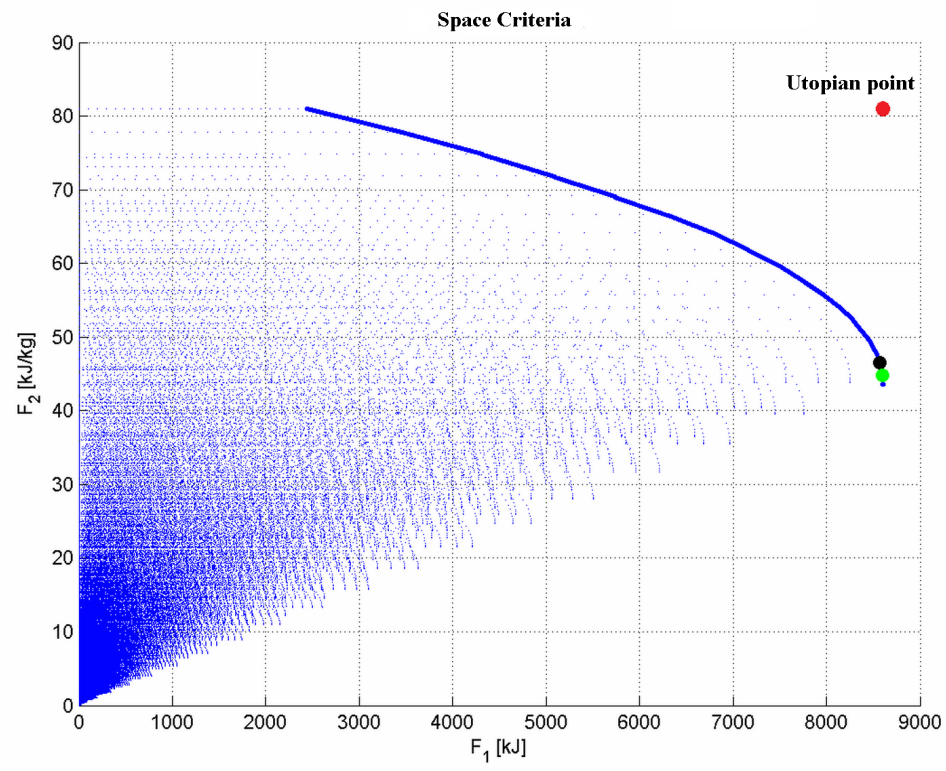

Fig. 9. Space criteria of optimal points marked

In this case, the utopian point was:

$F^{u}=[8599,187,7]^{T}$

The optimal points in both metrics are as follows:

- $F^{e}=[8593.445 .0]^{T}$ (green point on the graph),

- $F^{c}=[8570.546 .4]^{T}$ (black point on the graph).

In the space of decision parameters correspond to the points:

- $X^{e}=[0.20 .0320 .22094]^{T} \mathrm{mmmrev} . / \mathrm{s}$,

$-X^{c}=[0.20 .0480 .22094]^{T}$ mmmrev./s.

\section{Summary}

This work presents an analytical approach to the problem of optimizing the shape of the flywheel. This approach can significantly reduce the computational complexity of the solution (e.g., as compared to the Finite Element Method), but is suitable for use only for the shape of a simple geometry.

The method of selection of the optimal solution takes into account two parameters: the moment of inertia (stored energy) and energy density, none of them was identified as more 
important. Of course, you can choose a different method of scalarization, giving to one of the considered parameters more weight, which may lead to a different solution.

\section{References}

[1] Eschenauer Uwe.L., Hans A., Multiobjective Flywheel Design: A DOE-based Concept Exploration Task. ASME Design Engineering Technical Conferences, Sacramento, 1997.

[2] Piłat A., Introduction to COMSOL based modeling of levitated flywheel rotor. Stuttgart: COMSOL Conference, 2011.

[3] Marques M.I.L., Design and Control of an Electrical Machine for Flywheel Energy-Storage System, Lisbon: master thesis on Technical University of Lisbon, 2008.

[4] USF, Advanced composite flywhell - design example, 2007, http://www.weizmann.ac.il/ materials/Wagner/COURSES/CLASS $\ \% 2012 /$ Lecture $\backslash \% 2012 \_2007$.ppt.

[5] Genta G., Kinetic Energy Storage - Theory and Practice of Advanced Flywheel Systems, Butterworth-Heinemann Ltd, 1985.

[6] Kamf T., High speed flywheel design, Uppsala: Uppsala Universitet, 2012.

[7] Różewicz M., Modelowanie i optymalizacja kształtu koła zamachowego, Kraków: AGH bachelor thesis, 2012 supervisor: Adam Piłat Ph.D.

[8] MATLAB - documentation, 2014.

http://www . mathworks. com/help/optim/constrained-optimization.html

[9] Górecki H., Optymalizacja systemów dynamicznych. PWN Warszawa, 1993.

[10] Malte K., Secanell M., Mertiny P., Energy Storage in the Emerging Era of Smart Grids - chapter Rotor Design for High-Speed Flywheel. InTech, 2011. 